\title{
The influence of community opening on road traffic
}

\author{
Yumeng Cai \\ School of North China Electric Power University, Baoding 071003, China \\ 2469103191@qq.com
}

Keywords: Graph theory, Node degree variance, BPR impedance model

\begin{abstract}
This paper studies the influence of the cell is open to the surrounding road access, the establishment of a multi-index evaluation system first application of the basic comprehensive evaluation method of AHP, based on graph theory transportation network knowledge, in-depth analysis and select the network node of variance to evaluate the indicators for evaluating the impact on the surrounding roads open cell passage. Then, based on a problem created by the evaluation system to improve road BPR impedance model, further development of the mathematical model of vehicular traffic, in different scales on the next cell classification, quantitative analysis of the impact of open-cell, according to the final results of model from a traffic point of view prevailing rationalization proposals on the cell open.
\end{abstract}

\section{Introduction}

How to optimize the road network structure, improve the road capacity, improve the traffic situation and how to improve the efficiency of the open area. One view is that the closed area destroyed the structure of the urban road network, blocked the city "capillary", easy to cause traffic congestion. Some people think that this is related to the area, location, external and internal factors such as road conditions, can not be generalized. Urban planning and traffic management departments hope that we establish a mathematical model to study the impact of the open area on the surrounding roads, and provide quantitative basis for scientific decision-making.

\section{Traffic network evaluation model based on Analytic Hierarchy Process}

The analytic hierarchy process $(\mathrm{AHP})^{[1]}$ is especially suitable for the many factors that restrict the interrelated, forming complex and often lack of quantitative data, analysis of this kind of problem is decision-making and sort of a simple and practical method of multi criteria decision making. Because the evaluation indexes of road traffic network capacity are interrelated, restricted and difficult to quantify, AHP is used to sort the evaluation index.

\section{Establishment of hierarchical structure.}

When AHP is used to analyze the problem of road traffic network evaluation, firstly, the problem is organized and hierarchical, and a hierarchical structure model is constructed. To solve this problem, the target layer is the road network capacity index; the criterion layer for road network facilities evaluation index, traffic evaluation index; measures for road network layer grade, network density, road network traffic conditions etc.

\section{Structure judgment matrix.}

Let denote the road network grade, road network density, road space, $\mathrm{Z}$ road network traffic capacity, each taking two factors to express and $\mathrm{Z}$ on the ratio of the size of the result, with the judgment matrix A.

\section{Hierarchical single ordering and consistency checking.}

Determine the eigenvector of the $\mathrm{W}$ matrix A corresponding to the maximum eigenvalue of the normalized rank weights is the same level of the corresponding factors for a certain level of the relative importance of factors, namely single level sequencing using MATLAB to calculate the maximum eigenvalue of matrix A calculation, consistency index CI:

$$
\mathrm{CI}==0.06837
$$


Find the corresponding average random consistency index RI. As shown in Table 1. Table 1 The value of RI

\begin{tabular}{c|cccccccc}
\hline $\mathrm{n}$ & 1 & 2 & 3 & 4 & 5 & 6 & 7 & 8 \\
\hline $\mathrm{RI}$ & 0 & 0 & 0.58 & 0.9 & 1.12 & 1.32 & 1.41 & 1.45 \\
\hline $\mathrm{n}$ & 9 & 10 & 11 & 12 & 13 & 14 & 15 & \\
\hline $\mathrm{RI}$ & 1.46 & 1.49 & 1.52 & 1.54 & 1.56 & 1.58 & 1.59 & \\
\hline
\end{tabular}

According to the total order weight value selected measures evaluation indicators respectively road network grade, road network density, road network area connectivity, anti clogging ability, analysis of operating conditions of the road network, traffic intersection delay, saturation.

\section{The influence of each type of cell on the traffic convenience}

The BA road network traffic evaluation model ${ }^{[2]}$ based on the establishment of the problem based on the various types of residential road network traffic conditions using MATLAB software to analyze the quantitative effects before and after the open to traffic of various types of community.

The $\mathrm{BPR}^{[3]}$ function only takes into account the influence of the actual traffic on the travel time, and does not involve the non motor vehicle, pedestrian factors. In order to more accurately reflect the actual situation of the road impedance, the BPR function model is improved, and the characteristic of the road resistance function is determined by the characteristics of the new road.The parameters of each type of plot, chart analysis and node degree variance results are as follows:

(1) high density and low density residential external network: low density residential external network network structure node number were 100, 90; the internal node cell number is 10; (the initial node network, with each node into a new node s and $\mathrm{M}$ existing connection) in the parameter setting; under the condition of drawing high density and low density before and after external road network areas open road network simulation by MATLAB, as shown in Figure 1, traffic problems in the establishment of evaluation model to calculate the node degree based on variance as shown in Table 2.

Table 2 Node degree variance

\begin{tabular}{c|c|c}
\hline & $\begin{array}{c}\text { Low-density external road } \\
\text { network }\end{array}$ & $\begin{array}{c}\text { High-density external road } \\
\text { network }\end{array}$ \\
\hline \multirow{2}{*}{ Node degree variance } & 65.93 & 16.97 \\
\cline { 2 - 3 } & 50.69 & 102.41 \\
\hline
\end{tabular}

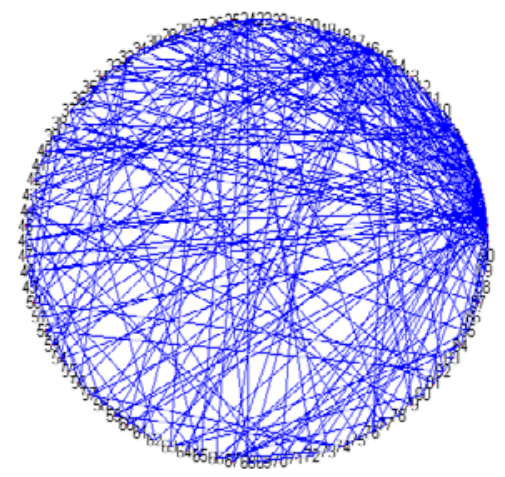

Simulation of high density external road network before opening

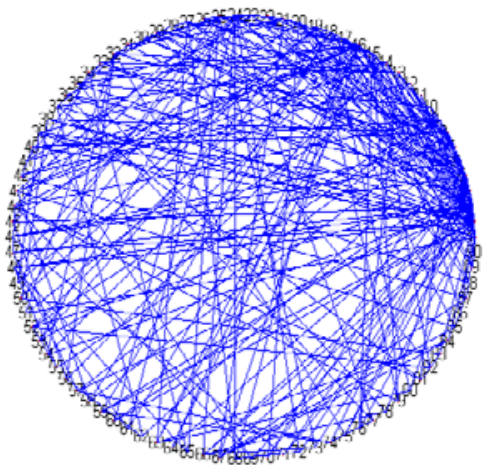

Simulation of low density external road network before opening 


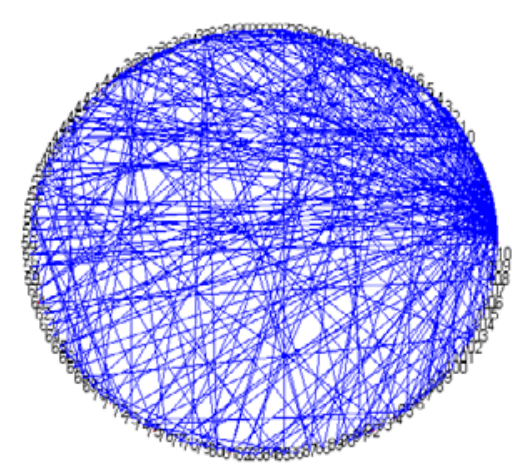

Simulation of high density external road network before opening

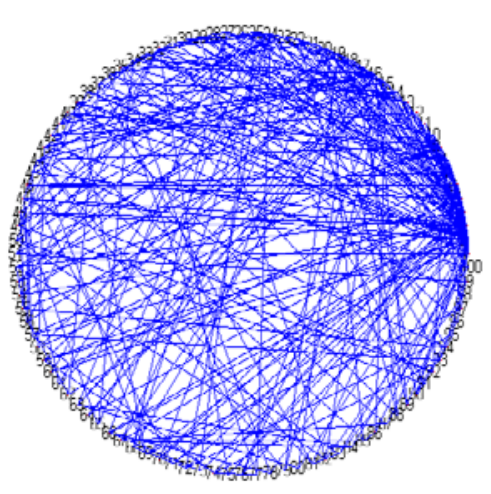

Simulation of low density external road network before opening

Figure 1

The comparison of the above four traffic network diagram, can visually analyze the complexity of the road traffic network after the opening of the district. According to the comparison of the two groups, it can be seen that the relationship between the degree of variance of the node density and the density of the external road network. Through theoretical analysis, when the access node starts from the maximum degree of nodes, the network degree variance increases with the increase in the number of access nodes, the network degree variance is greater than the initial network of variance, two large degree nodes connected to the road network structure increases unevenly, the connection node more multiplex network structure more uniform, more not to reduce the vulnerability of the network.

(2)Complex internal road network and simple internal road network: the number of nodes in urban road network is 100; the number of internal nodes in complex internal network and simple internal road network is 10 and 5, respectively.As shown in Figure 2.

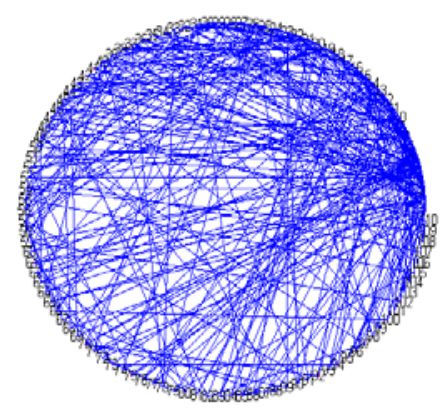

Open complex cell simulation

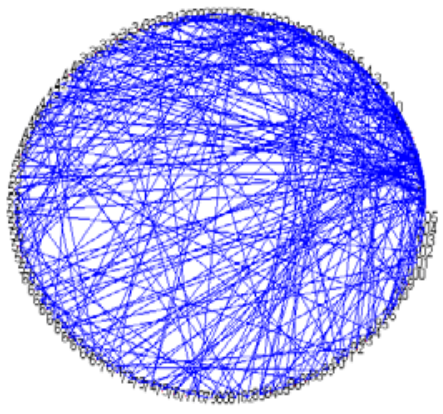

Open simple cell simulation Figure 2

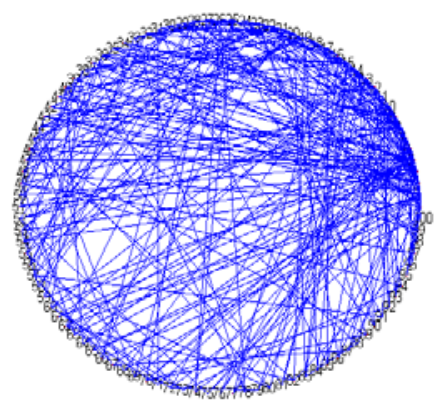

Not open cell simulation

After the opening of the complex internal network, the impact on the urban traffic network is greater. By comparing the variance of the node degree complex internal network nodes inside the network area than simple small degree variance value, analysis: when the cell network structure (similar to the average degree of nodes), the internal network structure is more complex (more nodes) is more obvious to the city traffic network vulnerability reduction.

\section{Rationalization proposals on cell open}

Based on the above model establishment and analysis process, we can put forward the following suggestions for urban planning and traffic management:

(1)Considering the road network vulnerability is as small as possible, the local area network and remote roads around the network should select both the conditions to allow for connection,nodes with smaller degree connection.. 
(2)Road construction planning departments can analyze the different area and location of the square area of the square for the convenience of the impact of the network traffic, in order to select the new roads and residential areas within the road transformation.

\section{Conclusions}

First, we use AHP to establish the basic evaluation model of road network capacity, and select several indicators with larger weight as the evaluation system in the evaluation index of urban road network. On this basis, we build a network node degree variance evaluation system from the point of view of the urban traffic network in graph theory.

Then, different types of residential areas are selected and constructed, and the effects of various types of residential areas on road traffic are compared with the established model. Considering the factors of the capacity of residential area, location, external and internal condition of the road road, construction of different types of cell, calculated before and after various types of community open road capacity, and quantitative analysis before and after various types of community open to traffic impact.

At last, based on the above model establishment and analysis process, we put forward some suggestions for urban planning and traffic management.

\section{References}

[1] Shoukui Si,Xijing Sun.Mathematical Modeling Algorithms and Applications.National Defence Industry Press.2015.

[2] Xiujuan Ma.Based on BA scale-free complex directed network evolution model[J].Electronic design engineering,.2012,16:11-13.

[3] Ning Liu, Shengchuan Zhao,Nan He.Research on road impedance function based on BPR function[J].Journal of Wuhan University of Technology (Transportation Science \& Engineering).2013,37(3):545-548. 